\title{
La obligación de comunicar resultados urgentes o inesperados de un estudio radiológico
}

\section{Cristian Garcia B..$^{\star}$}

1. Comité de Ética, Sociedad Chilena de Radiología. Santiago, Chile.

Años atrás, los radiólogos pensaban que su deber de comunicar resultados de los estudios no iba más allá de hacer su informe escrito. A veces, más por un asunto de cortesía y buena práctica médica, el radiólogo podía contactar al médico que solicitaba el examen y comunicarle los resultados cuando consideraba que los hallazgos radiológicos podían requerir un tratamiento inmediato, pero esto no era considerado obligatorio.

Posteriormente, en países como Estados Unidos, se determinó que los radiólogos tienen obligación legal de comunicar los hallazgos radiológicos a los médicos que solicitan los estudios $\mathrm{y}$, a veces, a los mismos pacientes en forma oportuna e inmediata si el cuidado del paciente lo requiere. Ante esto, las demandas por falta de comunicación contra radiólogos han ido en ascenso. Asimismo, han aumentado también las demandas contra los médicos clínicos que solicitan un estudio y no consultan precozmente al radiólogo por el resultado y esto determina consecuencias negativas para el paciente.

\section{¿Es realmente el deber del radiólogo comunicar los hallazgos radiológicos al médico tratante? \\ Definitivamente la responsabilidad pro- fesional del radiólogo no termina después que ha hecho una interpretación minuciosa y un informe del examen. El radiólogo tiene obligaciones con los pacientes, aunque no}

los vea personalmente.

Puede no ser estrictamente necesaria la interacción directa con el paciente, pero no puede faltar la comunicación con el médico tratante cuando esto es necesario, en especial cuando puede estar en riesgo la salud del paciente.

De esta manera, se considera que todos los médicos involucrados en la atención de un paciente, tienen algún grado de responsabilidad en su atención de salud, incluyendo los radiólogos.

Los radiólogos deben comunicar los hallazgos de un estudio al médico referente cuando descubren un hallazgo radiológico que requiere inmediata atención. La recepción oportuna del informe es más importante que el método de entrega y la comunicación de la información es tan efectiva como el sistema que transmite la información.

Cuando el radiólogo no puede localizar al médico que solicitó un examen y considera que es necesario un tratamiento inmediato del paciente, debe comunicárselo directamente al paciente e informarle que debe acudir al Servicio de Urgencia para recibir atención.

Una vez que se completa la comunicación verbal, esto debe ser documentado con un mensaje al pie del informe o en la ficha clínica.

Es responsabilidad del radiólogo informar los estudios de la forma más rápida y eficiente posible, pero también es la responsabilidad del médico tratante y/o de su equipo, de leer 
y tomar en cuenta los hallazgos del informe de la manera más rápida y eficiente posible.

El médico tratante es también responsable de incluir en la solicitud del examen la información clínica relevante y una hipótesis diagnóstica. Con esto mejora el rendimiento y la relevancia clínica del informe y el beneficiado final es el paciente.

El Código de ética médica de la American Medical Association establece que "los valores éticos y los principios legales suelen estar estrechamente relacionados, pero las obligaciones éticas generalmente superan los deberes legales". Es claro entonces, que, desde el punto de vista de la ley, se requiere que los radiólogos comuniquen los hallazgos importantes a los médicos de referencia de una manera que garantice razonablemente la recepción o alternativamente a los pacientes mismos.

Desde la perspectiva de un imperativo ético o moral, los médicos deberían querer hacer estas comunicaciones, al margen del aspecto jurídico o del temor a una eventual demanda.

\section{¿Es necesario precisar qué hallazgos radiológicos pueden requerir de atención inmediata y deben ser comunicados a los médicos tratantes?}

Algunas instituciones han intentado crear listas de diagnósticos que eventualmente pueden requerir una atención médica precoz y que obligan al radiólogo a comunicar los resultados al médico tratante. Sin embargo, estas listas pueden no ser prácticas y eventualmente perjudiciales desde una perspectiva jurídica.

Se asume que el Radiólogo, como todo médico formado, no requiere una lista para definir si un hallazgo radiológico es urgente, grave o adverso para la salud de un paciente. Los radiólogos son capaces de juzgar por sí mismos cuando un hallazgo requiere especial atención.

De acuerdo a los criterios del ACR, las situaciones que pueden justificar una comunicación directa y rápida con el médico tratante incluyen las siguientes:

a) Hallazgos que sugieren la necesidad de una intervención inmediata o urgente: en la mayor parte de los casos, esto puede ocurrir en los Servicios de Urgencia, Unidades de tratamiento intensivo.

b) Hallazgos que discrepan con una interpretación anterior de un mismo examen y donde la falta de un tratamiento puede afectar negativamente la salud del paciente.

c) Hallazgos que el radiólogo considere eventualmente graves para la salud del paciente que, si bien pueden no requerir atención inmediata, si no reciben tratamiento, pueden empeorar con el tiempo y llevar a un resultado adverso para el paciente: procesos infecciosos agudos, posibles lesiones malignas, etc.

\section{¿Es correcto hacer un informe preliminar?}

Cuando es necesario, un informe preliminar puede preceder al informe final, con el fin de acelerar la atención del paciente. Puede contener información limitada o incompleta y comunicarse por escrito, por vía electrónica o verbalmente y debe quedar documentado.

Debe ser seguido lo antes posible del informe definitivo y se debe informar cualquier variación significativa en los hallazgos $\mathrm{y} / \mathrm{o}$ conclusiones entre las interpretaciones preliminares y finales de tal manera que garantice la recepción por parte del médico encargado del tratamiento. La documentación de la comunicación de cualquier discrepancia debe incorporarse en el informe final.

Desde el punto de vista jurídico, estas comunicaciones deben documentarse, idealmente en el informe de radiología o el registro médico del paciente y puede incluir el tiempo, el método de comunicación y el nombre de la persona a quien se entregó.

Aunque se pueden considerar otros métodos de comunicación, como mensajes de texto, mensajes de voz, correo electrónico y otros métodos no tradicionales, estos no necesariamente garantizan la recepción de 
la información. Estas pueden además no respetar las normas de privacidad del paciente.

\section{¿Y las comunicaciones informales?}

Las comunicaciones informales, que pueden ocurrir en un pasillo, en una reunión clínica, etc, se pueden utilizar para tomar decisiones sobre el tratamiento, pero conllevan un riesgo inherente al no contar con estudios de comparación, informes previos y pueden ocurrir en condiciones de visión subóptima. Lo mismo puede ocurrir también al interpretar de manera informal estudios externos, por lo que recomendable establecer un sistema de informe formal en estas instancias.

\section{Lectura recomendada}

1. Berlin L. Malpractice Issues in Radiology. Communication of the Urgent Finding. AJR 1996; 166: 513-515.

2. The Royal College of Radiologists. Standards for the communication of critical, urgent and unexpected significant radiological findings. Second edition, 2012. En: https://www.rcr.ac.uk/sites/default/files/docs/radiology/ pdf/BFCR\%2812\%2911_urgent.pdf, Consultado el 29 de junio de 2019.

3. The American College of Radiology. ACR Practice parameter for communication of diagnostic imaging findings, 2014. En: https://www.acr.org/-/media/ACR/ Files/Practice-Parameters/CommunicationDiag.pdf. Consultado el 30 de junio de 2019.

4. Berlin L. Communicating radiology results. Lancet 2006; 367: 373-375.

García C. La obligación de comunicar resultados urgentes o inesperados de un estudio radiológico. Rev Chil Radiol 2019; 25(2): 44-46.

*Correo electrónico: Cristián García / cgarciabruce@gmail.com 